\title{
¿Desarrollo sostenible o sustentable? La controversia de un concepto
}

\section{Sostenible or sustentable development? The controversy of a concept}

\author{
Jaime Ernesto Rivera-Hernández ${ }^{1}$ \\ Centro de Estudios Geográficos, Biológicos y Comunitarios, \\ Sociedad Civil, México \\ Napoleón Vicente Blanco-Orozco² \\ Universidad Nacional de Ingeniería, Nicaragua
}

\author{
Graciela Alcántara-Salinas ${ }^{3}$ \\ Centro de Estudios Geográficos, Biológicos y Comunitarios, \\ Sociedad Civil, México \\ Eric Pascal Houbron ${ }^{4}$ \\ Universidad Veracruzana, México
}

\author{
Juan Antonio Pérez-Sato 5 \\ Colegio de Posgraduados, México
}

\begin{abstract}
Resumen. Se presenta una revisión y discusión de los conceptos desarrollo sostenible y desarrollo sustentable, en el afán de aclarar su origen y dilucidar si los conceptos son o no sinónimos y si ambos son correctos y válidos. Se incluye un breve panorama de cómo se han usado ambos conceptos tanto en la literatura científica, como en textos y leyes gubernamentales y de divulgación; también se describe la controversia que se ha generado por el uso no diferenciado de estos términos y las corrientes de pensamiento que los sustentan. Se concluye que, a pesar de que lingüísticamente ambos términos son correctos y sinónimos casi perfectos, representan posturas e ideologías diferentes, ya que el "desarrollo sostenible", representa un enfoque principal sobre el desarrollo y busca la manera que sea sostenido por medio del cuidado del ambiente y el "desarrollo sustentable", enfatiza el cuidado de los recursos naturales y su uso, pero mediante un modelo que proteja el ambiente como prioridad. .
\end{abstract}

Palabras clave. Desarrollo, sostenible, sustentable, términos, controversia

\begin{abstract}
A brief revision regarding "sostenible" and "sustentable" development is shown, with the objective of clarify their origin, and if these concepts are synonymous or not, and whether both are correct and valid. A brief outlook about how these concepts have been used in scientific, governmental and popular literature is included, and also, the controversy generated regarding the not differentiated use between these terms is described. We conclude that, although linguistically speaking both terms are correct and are synonymous, both terms represent different ideologies, since the term "sostenible" keep focus on the "development"; and in the term "sustentable", the focus is on natural resources protection and management, but with a model that prioritise the protection of environment.
\end{abstract}

Keywords. Sustainable, development, sostenible, sustentable, concept, controversy

\footnotetext{
${ }^{1}$ Jaime Ernesto Rivera-Hernández. Centro de Estudios Geográficos, Biológicos y Comunitarios, Sociedad Civil (GEOBICOM, S.C.), Veracruz, México. Doctor en Ciencias Naturales para el Desarrollo, DOCINADE-Costa Rica. Dirección electrónica: jriverah@geobicom.org

${ }^{2}$ Napoleón Vicente Blanco-Orozco. Facultad de Electrotecnia y Computación, Universidad Nacional de Ingeniería, Managua, Nicaragua. Doctor en Ciencias Naturales para el Desarrollo, DOCINADE-Costa Rica. Dirección electrónica: blanconapoleon@yahoo.com

${ }^{3}$ Graciela Alcántara-Salinas. Centro de Estudios Geográficos, Biológicos y Comunitarios, Sociedad Civil (GEOBICOM, S.C.), Veracruz, México. Doctora en Etnobiología, Universidad de Kent, Reino Unido. Dirección electrónica: graasrivalcan@gmail.com

${ }^{4}$ Eric Pascal Houbron. Universidad Veracruzana, Veracruz, México. Doctor en Ingeniería de Procesos Industriales, Instituto Nacional de Ciencias Aplicadas, Francia. Dirección electrónica: ehoubron@uv.mx

5Juan Antonio Pérez-Sato. Juan Antonio Pérez-Sato. Maestría en Paisaje y Turismo Rural, Colegio de Postgraduados, Campus Córdoba. Dirección electrónica: pantonio@colpos.mx
} 


\section{Introducción}

Los términos "desarrollo sostenible", "desarrollo sustentable", "sostenibilidad" y "sustentabilidad" son utilizados indistintamente en diferentes medios de comunicación como publicaciones científicas, libros académicos, textos gubernamentales, leyes, revistas y artículos de divulgación, periódicos, internet, películas, radio y televisión; así mismo, son usados cotidianamente en el vocabulario de especialistas en áreas como la economía, turismo, biología y en otras ciencias ambientales y también en aspectos relacionados con el desarrollo. Ejemplo de lo anterior son los artículos científicos de López, López y González (2007), con el título "Una experiencia chontal, Desarrollo Rural Sustentable", o el de Vilches, Gil y Cañal (2010), denominado "Educación para la sostenibilidad y educación ambiental". En el ámbito gubernamental, podemos citar a la Ley General de Desarrollo Forestal Sustentable de México $(\mathrm{H}$. Congreso de la Unión, 2003).

Tomando en cuenta lo anterior, es importante tener claridad en el origen de estos conceptos, que se han venido utilizando como sinónimos, sobre todo cuando estas palabras se mencionan en contextos de crecimiento, mejora o desarrollo. En este ensayo pretendemos dejar en claro el origen de estos conceptos, su validez para los países de habla hispana, con base en la Real Academia de la Lengua Española (RAE), que es la institución que se encarga de la regulación lingüística y los cambios que experimenta la lengua española (RAE, 2014a). La aclaración de estos términos para los hispanohablantes es crucial sobre todo cuando encontramos tales definiciones en otros idiomas como el inglés, italiano o portugués y, principalmente, con la controversia generada por el uso indistinto de estos conceptos en español; además, es importante determinar cuál es el término correcto y el que debería utilizarse, o bien, dejar clara la definición de cada uno de ellos en el caso de que se trate de conceptos válidos con definiciones diferentes, lo que se pretende lograr en este artículo por medio de una revisión y análisis de la literatura especializada.

\section{El origen}

La preocupación por el ambiente y su sostenibilidad o sustentabilidad inicia como una preocupación ambientalista y el punto de partida, parece corresponder a los siguientes momentos históricos: a) la fundación de la Unión Internacional para la Conservación de la Naturaleza (UICN) en 1948, para contribuir a encontrar soluciones prácticas para los principales desafíos ambientales y de desarrollo que enfrentaba ya el planeta; b) en 1955, se celebra un coloquio denominado "Man's role in Changing the Face of the Earth", que analizó el papel del ser humano en la transformación de la superficie terrestre (Gracia-Rojas, 2015) y c) la publicación de Carson (1962) "Primavera silenciosa", cuyo libro contribuyó a la generación de una nueva conciencia ambiental de escala global (Barrios et al., 2007).

$\mathrm{El}$ origen y los antecesores de estos conceptos se remontan a la década de los 70 (Sheinbaum, 2007), cuando se manifestó la crítica a la viabilidad del crecimiento económico como objetivo condicionante del desarrollo; en esta época, se acuñó el concepto de Ecodesarrollo; Maurice Strong del Programa de las Naciones Unidas para el Desarrollo (PNUMA), lo formuló primero, aunque posteriormente Ignacio Sachs lo elaboró y le dio difusión internacional (Riechmann, 1995); este concepto surge con el fin de armonizar el aumento de la producción, que urgentemente requerían los países del Tercer Mundo, con el respeto necesario a los ecosistemas, a fin de conservar las condiciones de habitabilidad de la Tierra (Naredo, 1996). Esta propuesta significó una crítica al modelo de desarrollo basado en el crecimiento económico sin respeto del ambiente y sin un componente de justicia social e intergeneracional. 
No obstante lo anterior, luego se inició una diferenciación de concepciones cuando la diplomacia estadounidense manifestó su desaprobación del concepto de Ecodesarrollo (Naredo, 1996; Sachs, 1994), promulgando lo inapropiado del término sobre todo en documentos vinculantes de organismos internacionales como la Organización de las Naciones Unidas (ONU); de esta manera, este término fue sustituido más tarde por el de "desarrollo sostenible", expresión que se podía aceptar por parte de los economistas que fundamentaban teóricamente el modelo de desarrollo "occidental y capitalista" predominante entonces, al confundirse con el concepto de "desarrollo dutosostenido" (self sustained growth), introducido tiempo atrás por Rostow y muy empleado por economistas que teorizaban el desarrollo desde el punto de vista de la ciencia económica (Naredo, 1996).

En 1980, en la Primer Estrategia Mundial de la Conservación, elaborada por la Unión Internacional para la Conservación de la Naturaleza (UICN), con la asesoría, cooperación y el apoyo financiero del Programa de las Naciones Unidas para el Medio Ambiente (PNUMA) y el Fondo Mundial para la Naturaleza (WWF), en colaboración con la Organización de las Naciones Unidas para la Agricultura y la Alimentación (FAO) y la Organización de las Naciones Unidas para la Educación, la Ciencia y la Cultura (UNESCO), se utiliza por primera vez el término "aprovechamiento sostenible" que propone tres objetivos generales: a) mantener los procesos ecológicos esenciales y los sistemas vitales, b) preservar la diversidad genética y c) asegurar el aprovechamiento sostenible de las especies y los ecosistemas. Este documento toma el modelo de sostenibilidad de la biología de poblaciones.

No fue hasta la aparición del documento Nuestro futuro común, también conocido como Informe Brundtland, cuando surge y se define formalmente el término en inglés sustainable de- velopment (ONU, 1987). En dicho texto, el término se entiende como: "el desarrollo que satisface las necesidades de la generación presente sin comprometer la capacidad de las generaciones futuras para satisfacer sus propias necesidades" (ONU, 1987, p.23). Sin embargo, el concepto significa una definición ética inicial, lo cual permite conciliar las diferencias existentes entre los diversos actores tanto de la sociedad civil, como gubernamentales, reconociendo además las diferencias entre naciones y cómo se acentúan estas a causa de las deudas externas de los países en vía de desarrollo.

Dicho informe busca establecer un espacio de convivencia para una política de consenso que sea capaz de disolver las diferentes visiones e intereses entre las naciones, sus pueblos, su sociedad civil y otros actores que conforman el complicado mundo del desarrollo sostenible o sustentable. Empero, este primer concepto de Sustainable development en vez de considerar un cambio de fondo, retoma y refuerza el paradigma tradicional de desarrollo (Barrios et al., 2007). Es también importante mencionar que en el Informe Bruntdland se refleja la discusión que en ese momento está teniendo lugar sobre el desarrollo sostenible y trata de explicar el sentido que se le daba al adjetivo "sostenible".

\section{La controversia}

Actualmente se utiliza sin distinción los términos: sostenible, sustentable, duradero o perdurable. En inglés existe solo el término "sustainable" y en francés "perdurable o durable"; en español se utilizan los términos sostenible y sustentable, lo que ha generado discusiones. La confusión del concepto tiene su génesis en el proceso de traducción del idioma inglés al español, como sostenible o sustentable: se utilizan ambos términos para lo que aparentemente es el mismo concepto. Según Flores (2008), por un lado el vocablo "sostenible" tiene su origen en la palabra "sostenido", cuyo significado es que algo puede 
mantenerse por un tiempo determinado; por otro lado, "sostenible" significa que algo se puede mantener por tiempo indefinido, sin que colapse o se deteriore. Por su parte, "sustentable" se origina del verbo "sustentar', cuyo significado se enfoca hacia un proceso independiente, que no necesita de recursos externos para mantenerse.

$\mathrm{Al}$ revisar estos conceptos en el Diccionario de la Lengua Española (RAE, 2014b), encontramos sus definiciones, las cuales se citan a continuación:

\begin{abstract}
"Sustentable. Que se puede sustentar o defender con razones."

"Sostenible. Dicho de un proceso que puede mantenerse por sí mismo, como lo hace, por ejemplo, un desarrollo económico sin ayuda exterior ni merma de los recursos existentes. Que se puede sostener (opinión, situación). Especialmente en Ecología y Economía, que se puede mantener durante largo tiempo sin agotar los recursos o causar grave daño al medio ambiente."

"Sostenibilidad. Cualidad de sostenible."

"Sustentabilidad. Estapalabranoexisteen eldiccionario, es decir, no es aceptada como válida."
\end{abstract}

Es primordial apuntar que la condición de Sostenibilidad se refiere al equilibrio de una especie con los recursos de su entorno, por lo que este término tiene su origen en la biología de poblaciones, especialmente utilizado en temas de pesquería y forestería (Zúñiga, 2011).

$\mathrm{Al}$ analizar las definiciones de los vocablos en cuestión, hay que puntualizar que el término "sostenible" se encuentra más desarrollado y enfocado hacia lo que es el sustainable development, en cambio, "sustentable" se refiere más a una idea, un concepto o un punto de vista. Debe remarcarse la ausencia del término "sustentabilidad" en el Diccionario de la Lengua Española. En adición, al continuar analizando las diferencias desde el punto de vista lingüístico, se toma en cuenta lo referido por Márquez (2000) y por la Real Academia de la Lengua Española (2014b), se menciona que ambos términos son adjetivos verbales o postverbales, es decir, que derivan de los verbos "sostener" y "sustentar" respectivamente y que pertenecen al tipo de adjetivos que se forman mediante el agregado del sufijo -able o -ible a la raíz del verbo, como por ejemplo: amar-amable, o temer-temible. El sufijo -ble indica capacidad o aptitud para recibir la acción del verbo, es decir, "sostenible" es lo que es "capaz de sostener o de sostenerse" y "sustentable" aquello que es "capaz de sustentar o sustentarse'. De esta manera, se puede afirmar que el "desarrollo sostenible" es "el desarrollo que puede o es capaz de sostenerse" y el "desarrollo sustentable" es "el que puede o es capaz de sustentarse'.

Tomando en cuenta lo anterior, se puede afirmar que, gramaticalmente, el uso de ambos adjetivos como calificativos del sustantivo "desarrollo" es válido. Sin embargo, desde el punto de vista del significado, se debe investigar si los dos son igualmente válidos o sólo uno de ellos lo es. Para esclarecerlo hay que volver a las definiciones del Diccionario de la Real Academia Española (2014b), en que "sostener" es: "Sustentar, mantener firme algo". En cuanto a "sustentar", el mismo diccionario lo define como: "sostener algo para que no se caiga o se tuerza". De esta manera, el diccionario de la RAE establece estos verbos como sinónimos casi perfectos, por lo que, observados desde esta perspectiva, forzosamente se debe concluir que, tomando en cuenta estrictamente el lenguaje, el léxico y la semántica, los verbos "sostener" y "sustentar" son sinónimos y, por tanto, su uso es indistinto (Márquez, 2000).

Por otra parte, hablando del sustantivo "sustentabilidad", el hecho de que no figure en el diccionario de la RAE no quiere decir que no exista tal término, pues el diccionario no determina la existencia o inexistencia de ningún vocablo, ya 
que se trata de derivaciones lógicas y naturales, que en algunos casos pueden estar como latentes, como esperando que alguien los emplee para materializarse. De modo que, cuanto esto ocurre, no puede hablarse de la creación o invención de una nueva palabra, sino de extraerla de un reservorio natural. Un buen ejemplo es el sustantivo "gobernabilidad", que es a su vez un derivado natural del adjetivo "gobernable". La existencia de dicho sustantivo, aunque no esté en el diccionario, es de una lógica abrumadora, ya que inevitablemente el verbo "gobernar" genera un adjetivo derivado que es "gobernable" y este, por su parte, genera también necesariamente un sustantivo derivado, que es "gobernabilidad" (Márquez, 2000).

Adicionalmente, al revisarse la literatura científica actual se encuentra que el uso de ambos términos es indiscriminado, así como que muchos otros autores prefieren el uso de uno $u$ otro término y hay quienes prefieren el uso de "sostenibilidad" o "sustentabilidad" con el fin de eliminar el término "desarrollo" de su significado. Por ejemplo, Gallopín (2003) opina que el concepto de "desarrollo sostenible" difiere de lo entendido por "sostenibilidad", iniciando con entender que la palabra "desarrollo" conduce a una idea de cambio gradual y direccional; por el contrario, "sostenibilidad" indica el mantenimiento de una situación existente de un estado o sistema.

De la misma manera, Riechmann (1995) menciona que "desarrollo" (tal como se define habitualmente) y "desarrollo sostenible", más que conceptos emparentados, son antagónicos; sostiene que si resulta imposible imponer la visión ecologista del desarrollo sostenible sobre la engañosa interpretación productivista, tal vez lo mejor sea abandonar este término pues no se trata de salvar la idea convencional de "desarrollo", sino más bien de avanzar hacia la nueva noción de sustentabilidad.
Al revisar la historia reciente del concepto "desarrollo sostenible", después de su primera aparición en el informe Brundtland en 1992, se produce la Cumbre de Río (Cumbre de la Tierra), donde se proclamó el desarrollo sostenible como una nueva meta de la humanidad para superar la crisis ambiental global. Por lo anterior, este término se popularizó como un objetivo social supuestamente deseado, pero a la vez se volvió un concepto controversial, ya que, por otro lado, se comenzó una tendencia de pensamiento la cual definía que, para conseguir este desarrollo sostenible era necesario continuar con el crecimiento económico, medido según los parámetros economicistas de los países ricos, por lo que también en la Cumbre de Río se reconocieron ampliamente las limitaciones ambientales de este concepto.

De esta manera, el crecimiento asociado al desarrollo es un crecimiento económico constante y regular en el tiempo, sin altibajos desequilibradores y, además, olvida cualquier contenido ecológico (García y Priotto, 2009). Por esto algunas organizaciones latinoamericanas han querido diferenciar su posición de la posición oficial (la de las Naciones Unidas y los gobiernos), adoptando el término sustentable. De hecho, en Argentina, México y Chile se utiliza con mayor frecuencia el término "desarrollo sustentable", pero sin que haya quedado claramente definida la diferencia (Flores, 2008).

A partir de la presentación del concepto por parte de la Comisión Mundial sobre el Medio Ambiente y Desarrollo (WCED) en el informe Brundtland y después en la Cumbre de Río, se puede notar el uso distinto de los conceptos sostenible y sustentable. Por ejemplo, en el manifiesto por la vida "Por una Ética para la Sustentabilidad" (PNUMA, 2002), se plantea que: "la ética de la sustentabilidad debe partir del reconocimiento de los límites y potenciales naturales y culturales para el desarrollo sustentable" (p. 3) 
y también menciona que "reconociendo los límites de la naturaleza, puede ayudarnos a definir una nueva visión y una nueva comprensión del mundo para enfrentar los enormes desafíos de la humanidad" (p. 1) y que: "debemos cambiar las concepciones dominantes de la racionalidad económica y tecnológica que intentan legitimar procesos de dominación, insustentabilidad ecológica e inequidad social" (p. 1). El desarrollo sostenible, al contrario de lo sustentable, se plantea como concepto dual y confuso que revive el desarrollismo y promueve la falacia de un crecimiento económico ilimitado sobre la naturaleza limitada del planeta y se convierte entonces, en lo que alguna vez se ha llamado un "concepto esencialmente discutible" (Riechmann, 1995). Este esquema de pensamiento es resultado del dominio filosófico del pensamiento económico de teóricos del desarrollo de los países del mundo industrializado de la época (Barrios et al., 2007).

La sostenibilidad responde a la clásica definición de lo sostenible del informe de Brundtland y se encuentra definida en un contexto donde se vincula el crecimiento económico con el uso de los recursos naturales. Lo sustentable es una concepción más integral que va mucho más allá de únicamente lo económico. Las corrientes latinoamericanas reconocen en el concepto de sustentabilidad una posición ética, como una manera de posicionarse frente a la vida y a los distintos elementos del ambiente; es más un juego de armonización de éticas y racionalidades, con el componente de la cultura latinoamericana (Barrios et al., 2007; Leff, 2006).

La ética del desarrollo sustentable difiere del planteamiento de desarrollo sostenible, el cual trata de armonizar las peculiaridades del mercado con los intereses del Estado y del ciudadano común; por el contrario, la ética de lo sustentable implica la necesidad de conjugar una serie de principios básicos dentro de una ética del bien común, sin recurrir a la supuesta racionalidad económica e instrumental que se plantea como modelo que debe incorporar el ser humano moderno y que son tales principios, una antítesis del propósito de la sustentabilidad.

El desarrollo basado en lo sostenible del crecimiento económico se ha fundamentado en paradigmas y conductas irreflexivas y en comportamientos insustentables que se basan en los principios del individualismo y de la competencia de la racionalidad económica.

Por lo tanto, el desarrollo sustentable requiere una moral distinta ante el consumo y una ética de la frugalidad; se habla entonces de un nuevo modo de producción fundado en los potenciales de la naturaleza y de la cultura y no en las leyes teóricas y pragmáticas del mercado. La ética del desarrollo sustentable promulga respeto a la diversidad, a la diferencia, a la otredad y a la alternativa. El objetivo es la refundación de una nueva concepción de racionalidad productiva sustentada en una nueva economía con principios ecológicos, morales y culturales como condición de sustentabilidad (Leff, 2006).

El nuevo enfoque de desarrollo implica, pues, considerar principios de ética para la sustentabilidad que se expresen en valores de solidaridad de la humanidad para con el planeta, lo primordial es el "ser" antes de la posesión material y, principalmente, dar un lugar primordial a la distribución de los recursos de la naturaleza antes del crecimiento, así como utilizar como práctica la gestión colectiva de bienes comunes de la naturaleza; en otras palabras, el nuevo concepto de sustentabilidad se fundamenta en una ética que implique impulsar la solidaridad, reciprocidad y el bien común por encima del principio egoísta de producir ganancias, riquezas y comodidad basado en un acceso privilegiado a los recursos naturales (PNUMA, 2002).

Por consiguiente, la diferencia de los términos, la cual va mucho más allá de una mala traduc- 
ción del inglés, ahora es producto de los avances y planteamientos que desde las corrientes ambientalistas han establecido una discrepancia entre los dos términos. El discurso del "desarrollo sostenible" parte de una idea de que sus políticas buscan armonizar el proceso económico con la conservación de la naturaleza; favorece un balance entre la satisfacción de necesidades actuales y las de las generaciones futuras; sin embargo, se pretenden cumplir los objetivos revitalizando el viejo mito desarrollista, promoviendo un crecimiento económico sostenible sobre la naturaleza limitada del planeta; es decir, lo sostenible le da prioridad al crecimiento económico sobre la protección de la naturaleza (Barrios et al., 2007).

El desarrollo sostenible como conceptualización armónica entre lo antropológico y lo ecológico se ha cubierto de una mayor elaboración epistemológica. Se puede, entonces, enfocar el concepto como un desarrollo que implique el despliegue y la adquisición de capacidades humanas en conjunto con el respeto de la sostenibilidad ecológica como un principio básico de justicia intergeneracional (Arribas, 2007), que a su vez implique la posibilidad de convivir en el entorno conociendo sus demarcaciones. Por lo que el desarrollo sostenible se convierte en sustentable si logramos vivir dentro de los límites de los ecosistemas (Riechmann, 1995).

El desarrollo sustentable nos conduce a considerar varios elementos necesarios para su establecimiento, como reconocer la diversidad; de hecho, la sustentabilidad es una lucha por la diversidad ecológica y social en todas sus dimensiones (Barkin, 1998). Adicionalmente, la sustentabilidad se basa en la participación consciente de las personas en una lucha por justicia social, derechos humanos, diversidad cultural y respeto al ambiente, porque sin un cambio social y económico que signifique un nuevo pacto social de distribución de riquezas y de participación política y gobernanza democrática, no habrá un desarrollo sustentable, si acaso un desarrollo sostenido continuado al estilo occidental. Está, por ende, en la palestra, la definición de un desarrollo que indique un nuevo equilibrio entre el uso de la naturaleza y la atención a las necesidades humanas (Gudynas, 2004).

De esta manera, el desarrollo sustentable se debe pensar como una nueva relación con el ambiente, con un objetivo superior al equilibrio entre crecimiento económico y conservación de la naturaleza; se debe integrar el potencial ecológico, el desarrollo tecnológico, la cultura y la sociedad para construir un desarrollo sustentable que sea a la vez capaz de satisfacer las necesidades básicas de la humanidad, mejorando su calidad de vida. Se plantea una transformación productiva, de valores éticos y sociales, con el fin de construir un nuevo modelo de interactuar con la naturaleza (Gudynas, 2004).

\section{Conclusión}

La discusión en torno al desarrollo sustentable o sostenible, no sólo tiene que ver con las diferencias o similitudes semánticas que ya hemos discutido líneas arriba o a una depuración terminológica académica, sino que tiene implicaciones más profundas, de índole social, demográfica, cultural, valorativa y sobre todo política, debido a que tiene que ver directamente con la existencia de la vida del ser humano y su entorno (Rodríguez y Govea, 2006).

En términos generales, podemos mencionar que la confusión inicia con el hecho de que el vocablo anglosajón "sustainable" fue traducido indistintamente como "sustentable" o "sostenible". Según la Real Academia de la Lengua Española (2014), el término "sostenible" y "sostenibilidad" son los correctos, o al menos, son los más adecuados a la idea original planteada en el informe Bruntland (ONU, 1987). Sin embargo, si se abaliza el significado de los verbos que dan 
origen a los términos en análisis, se observa que, al menos semánticamente, se trata de sinónimos casi perfectos. Por tal razón, la sociedad en general y los académicos en particular, han utilizado ambos términos indistintamente o han preferido el uso de uno sobre el otro, pero sin que la diferencia entre ellos quedara clara.

No obstante, al revisar la literatura especializada con respecto al tema, se puede concluir que los dos conceptos se han diferenciado por la posición ideológica que defienden, ya que mientras los países desarrollados conceptualizan un "desarrollo sostenible", que se mida en términos económicos para continuar con su modelo de desarrollo basado en reglas de mercado, el "desarrollo sustentable" plantea un cambio radical de la manera de uso para la producción, el consumo y la distribución de los recursos naturales.

De esta manera, la principal diferencia entre los términos en análisis, está en su uso para diferenciar posiciones de concepciones de desarrollo, ya que el término "desarrollo sostenible" es más utilizado por investigadores y profesionales de las ciencias sociales, económicas y políticas, en razón de que su enfoque o principal preocupación es el "desarrollo", el cual debe ser sostenido o sostenible para que el sistema productivo funcione, luego entonces para que el desarrollo pueda ser sostenible, debemos cuidar que los recursos naturales no se agoten.

En cambio, la visión de "desarrollo sustentable" es principalmente utilizado por investigadores y profesionales de las ciencias naturales, para quienes su principal preocupación $\mathrm{u}$ objetivo final es la conservación de los recursos naturales, mediante su uso racional y controlado; o sea, no se trata de no utilizar los recursos, sino de hacerlo de una manera que se garantice su conservación a futuro. Esta visión, como lo menciona Flores (2008), está encaminada a cambiar nuestros modos de producción, consumo y distribución de los recursos naturales y conlle- va a un nuevo diseño de las formas en las que comúnmente producimos, que causan una gran contaminación y, por lo tanto, destruyen el medio ambiente. Afirma Barkin (1988, p. 25) que "la sustentabilidad es un proceso más que un conjunto de metas bien específicas e implica una nueva forma de relacionarse con la naturaleza, la economía y la sociedad".

Por último, hay que tomar en cuenta la opinión de López, López y Ancona (2015), quienes mencionan que cuando alguien usa el vocablo "sostenible" se hace de acuerdo con el conocimiento que se posee, y señalan que no existe una sostenibilidad a largo plazo. Estos mismos autores apuntan que el término "sustentable" es utilizado como una forma de conciliar el crecimiento económico con el equilibrio de los ecosistemas, tratando de mantener una alta producción y proteger a la vez los recursos naturales, lo que es contradictorio si no se sabe cuánto se debe conservar y cómo hacerlo. Se concluye que lo más importante es que de todos depende que el desarrollo sea sostenido y sustentable y que los principios que originaron los conceptos sean aplicados.

Así, al referirse al desarrollo y asociarle el término "sustentable" debe entenderse que se está expresando un proceso de desarrollo equilibrado desde los puntos de vista social y económico y su interacción con el ambiente. Por su parte, el "desarrollo sostenible" se refiere a un proceso con características económicas que le permite al sistema perdurar en el tiempo.

Tomando en cuenta lo anterior, no es posible concluir si un término es mejor que el otro o si uno está mal utilizado, sino que cada uno responde a diferentes enfoques. Por lo tanto, dependiendo del enfoque y priorización, ya sea hacia el desarrollo (sostenible) o hacia la conservación de los recursos naturales (sustentable), se debe diferenciar su uso. Sin embargo, si ambas visiones se logran conjuntar y poner de acuerdo, tratando de encontrar un balance entre cuanto 
se pueden cuidar los recursos naturales sin descuidar el desarrollo humano o hasta que punto es posible tener un desarrollo sin afectar a los recursos naturales, entonces tendremos un modelo que produzca éxitos palpables sin importar si le llamamos desarrollo sostenible o sustentable.

Finalmente, al registrarse el término "sustentabilidad" en el diccionario de la RAE y darle una definición más correcta al término "sustentable" en el mismo diccionario, podríamos ampliar el uso de ambos términos y tal vez, podrían ser mejor entendidos y utilizados.

\section{Agradecimientos}

Se agradece mucho a los profesores del curso Desarrollo y Sostenibilidad, del Programa de Doctorado en Ciencias Naturales para el Desarrollo (DOCINADE): Willy Soto, Tomás Guzmán y Sayra Munguía, por la motivación para la realización del presente ensayo y por la revisión objetiva y los atinados comentarios del manuscrito final. Adicionalmente, se agradece la revisión y sugerencias para mejorar el texto de Feliza Ramón Farías y dos revisores anónimos.

\section{Referencias}

Arribas H., F. (2007). La idea de desarrollo sostenible. Sistema 196, 75-86. Recuperado de http:// www.ficad.org/lecturas/adicional_uno_unidad_tres_gads.pdf.

Barkin, D. (1998). Riqueza, pobreza y desarrollo sustentable. Editorial Jus y Centro de ecología y Desarrollo. Versión electrónica. México. Recuperado de http://www.eumed.net/libros-gratis/2005/db/db.pdf.

Barrios N., C., Castro A., U., Coria, L.G., González A., M., Martínez V., R. y Taddey D., L. (2007). La relación global-local. Sus implicancias prácticas para el diseño de estrategias de desarrollo. Red Académica Iberoamericana Local-Global-EUMEDNET. Recuperado de www.eumed.net/libros/2007a/259/index.htm.
Carson, R. (1962). Silent Spring. Boston: Ed. Houghton Mifflin..

Flores B., T. (2008) ¿Desarrollo sostenible o sustentable? Asociación Boliviana Prodefensa de la Naturaleza. Recuperado de https://www.google.com.mx/ url? sa $=$ t\&rct $=j \& q=\&$ esrc $=$ s\& source $=$ web \&c $\mathrm{d}=1 \& \mathrm{cad}=\mathrm{rja} \& \mathrm{uact}=8 \& \mathrm{ved}=0$ ahUKEwi D i J S r q L U A h X E 2 S Y K H U 9 W B C MQF g g r M A A \& u r l $=$ h t t p \% 3 A \% $2 \mathrm{~F} \% 2 \mathrm{~F} \mathrm{~s} \mathrm{a} 3 \mathrm{dc} 15 \mathrm{bc} 92033257$. jimcontent.com $\% 2$ Fdownload $\% 2$ Fversion $\% 2 \mathrm{~F} 1305553971 \% 2 \mathrm{Fmodule} \%$ 2F5154290770\%2Fname\%2Fdesarrollo\%2520 sostenible $\% 2520$ \% $\% 2520$ sustentable. pdf\&usg=AFQjCNG-v5RTsYFYiYY7oTN1UJraAe3Iwg\&sig=Iaq2FtmBHy1oWE4eE RW55g.

Gallopin, G. (2003). Sostenibilidad y desarrollo sostenible: un enfoque sistémico. CEPAL. Serie Medio Ambiente y Desarrollo Núm. 64. Naciones Unidas. Chile. Recuperado de http://repositorio.cepal.org/bitstream/handle/11362/5763/1/ S033120_es.pdf.

García, D. y Priotto, G. (2009). Educación Ambiental. Aportes Políticos y Pedagógicos en la construcción del campo de la Educación Ambiental. Secretaría de Ambiente y Desarrollo Sustentable. Buenos Aires, Argentina. Recuperado de http://www.campusvirtual.unt.edu.ar/file. php?file=\%2F284\%2FAportes_20Pol_C3_ADticos_20y_20Pedag_C3_B3cios.pdf.

Gracia, J.P. (2015). Desarrollo sostenible: Origen, evolución y enfoques. Documento de docencia No. 3. Bogotá: Ediciones Universidad Cooperativa de Colombia. 18 pp. Recuperado de https://revistas.ucc.edu.co/index.php/dodo/ article/view/1074.

Gudynas, E. (2004). Ecología, economía y ética del Desarrollo Sustentable. Coscoroba Ediciones. Centro Latino Americano de Ecología Social 
(CLAES) y Desarrollo, Economía, Ecología y Equidad-América Latina (D3E). 5a Edición. Montevideo, Uruguay. Recuperado de http://www.ecologiapolitica.net/gudynas/ GudynasDS5.pdf.

H. Congreso de la Unión. (2003). Ley General de Desarrollo Forestal Sustentable. Diario Oficial de la Federación, México. 25 de febrero, pp. 1-70. Recuperado de http://www.diputados.gob.mx/LeyesBiblio/pdf/259_240117. pdf.

Leff, E. (2006). Ética por la vida. Elogio de la voluntad de poder. Polis (5)13, 1-14. Recuperado de http://www.redalyc.org/ pdf/305/30551306.pdf.

López R., C.D., López H. y E.S. y González, E.J. (2007). Una experiencia chontal. Desarrollo rural sustentable. Trayectorias (9)24: 57-67. Recuperado de http://www.redalyc. org/pdf/607/60715115007.pdf.

López R., C.D., López H., E.S. y Ancona P., I. (2015). Desarrollo sustentable o sostenible: una definición conceptual. Horizonte Sanitario (4)2: 1-7. Recuperado de http://www. redalyc.org/pdf/4578/457845044002.pdf.

Márquez R., A. (2000, 5 de noviembre). Sostenible y Sustentable. Periódico El Nacional. Caracas, Venezuela.

Naredo, J.M. (1996). Sobre el origen, el uso y el contenido del término sostenible. Textos sobre sostenibilidad. Cuadernos de investigación urbanística, 41, 7-18. Recuperado de https://dialnet.unirioja.es/servlet/articulo?codigo $=1333758$.

ONU. (1987). Nuestro futuro común. Informe Brundtland. Comisión Mundial sobre el Medio Ambiente y el Desarrollo. Organización de las Naciones Unidas. Recuperado de http://www.un.org/es/comun/docs/?symbol=A/42/427.
PNUMA. (2002). Por una ética para la Sustentabilidad. Sétima Reunión del Comité Intersesional del Foro de Ministros de Medio Ambiente de América Latina y el Caribe. Programa de las Naciones Unidas para el Medio Ambiente. Oficina Regional para América Latina y el Caribe. Sao Paulo, Brasil.

Real Academia Española (2014a). Estatutos y reglamento de la Real Academia Española. Real Academia Española 300 Años. Madrid. Recuperado de http://www.rae.es/sites/default/ files/Estatutos_y_reglamento_nuevo.pdf.

Real Academia de la Lengua Española (2014b). Diccionario de la Lengua Española. Vigésima tercera edición. Colección de Diccionarios Espasa. España: Espasa. Recuperado de http://www.rae.es/.

Riechmann, J. (1995). Desarrollo Sostenible: la lucha por la interpretación. En: Riechmann, J., Naredo, J.M., Estevan, A. y Bermejo, R (eds). De la Economía a la Ecología. Madrid: Editorial Trotta. Recuperado de https:// sistemamid.com/panel/uploads/biblioteca/2014-09-30_10-56-06111186.pdf.

Rodríguez, I. y Govea, H. (2006). El discurso del desarrollo sustentable en América Latina. Revista venezolana de Economía y Ciencias Sociales (12)2: 37-63. Recuperado de http:// www.redalyc.org/pdf/177/17712202.pdf.

Sachs, I. (1994). Entrevista en Science, Nature. Societé, 2(3).

Sheinbaum, D. (2007). ¿Qué es la sustentabilidad? Recuperado de http://sepiensa.org.mx/ contenidos/2007/1_susten/susten1.html

UICN. (1980). Estrategia Mundial para la Conservación. La conservación de los recursos vivos para el logro de un desarrollo sostenido. UICN, PNUMA y WWF. 71 pp. Recuperado de https://portals.iucn.org/library/sites/library/ files/documents/WCS-004-Es.pdf. 
Vilches, A., Gil D. y Cañal, P. (2010). Educación para la Sostenibilidad y Educación ambiental. Investigación en la Escuela, 71, 5-15. Recuperado de http://www.uv.es/ vilches/ Documentos/EDS\%20y\%20EA.pdf.
Zúñiga, M.Y. (2011). Sostenibilidad y Sustentabilidad como estrategias para crear valor en las viviendas actuales. Tesina de Especialidad en Ingeniería Civil (Construcción). Facultad de Ingeniería. Ciudad de México: UNAM.

Recibido: 30 de mayo de 2017 Aceptado: 9 de junio de 2017 
